\title{
Stellschrauben mit großer Wirkung
}

Ansätze zur Gewinnung neuer Auszubildender in der Altenpflege

Lukas Slotala

$5.1 \quad$ Einleitung -72

5.2 Entwicklung der Ausbildungszahlen im Altenpflegebereich - 73

5.3 Maßnahmen zur Gewinnung neuer Auszubildender in der Altenpflegeausbildung - 75

5.3.1 WeGebAU-Förderung der Bundesagentur für Arbeit - 75

5.3.2 Bundeslandspezifische Ansätze - 76

5.4 Diskussion -78

5.5 Schlussbetrachtung und Ausblick auf das Pflegeberufegesetz - 80

Literatur - 81 


\section{- Zusammenfassung}

Der Beitrag analysiert ausgewählte Ansätze zur Gewinnung neuer Auszubildender in der Altenpflege. Zunächst wird die bundesweite Entwicklung der Ausbildungszahlen dargestellt. Darauf aufbauend werden verschiede Maßnahmen auf Bundes- und Länderebene zur Steigerung der Ausbildungsplätze in der dreijährigen Altenpflege analysiert. $\mathrm{Zu}$ diesen zählen die Umschulungsförderung der Bundesagentur für Arbeit, die Einführung einer Umlagefinanzierung sowie die Ansprache von Hauptschülerinnen und Hauptschülern. Abgewogen werden die Potenziale der Ansätze zur Erhöhung der Anzahl der Auszubildenden auf der einen Seite und Befürchtungen um eine Absenkung des Qualitätsniveaus in der Altenpflegeausbildung auf der anderen Seite. Der Beitrag schließt mit einem Resümee im Kontext des neuen Pflegeberufegesetzes.

The article analyses selected approaches to the recruitment of new trainees in geriatric nursing care. First, the development of trainee figures is illustrated. In a second step, the diverse measures at federal and regional levels to increase the number of places in three-year geriatric nursing care training are analysed. These comprise the re-training programme funded by the Federal Employment Agency, the introduction of pay-asyou-go financing as well as specific appeals to German secondary school leavers. The potentials of approaches to increase the number of trainees on the one hand are balanced against concerns regarding a lowering of quality standards in geriatric nursing care training on the other hand. The article concludes with a summary in the context of the recent Nursing Professions Reform Act.

\subsection{Einleitung}

Unter den Branchenvertretern der ambulanten und stationären Langzeitpflege sowie über politische Parteigrenzen hinweg haben die Sorgen im Zusammenhang mit dem sich aktuell zuspitzenden Personalmangel im dreijährigen Altenpflegeberuf (BA 2018) deutlich zugenom- men. Vor dem Hintergrund eines prognostisch weiterwachsenden Personalbedarfs als Konsequenz des demografischen Wandels (Hackmann et al. 2016; Kochskämper 2018; Rothgang et al. 2012; Ostwald et al. 2010) avanciert die Suche nach vielversprechenden Ansätzen zur Gewinnung neuer Auszubildender in der Altenpflege zur prioritären Aufgabe der Gegenwart und Zukunft.

In der jüngsten Vergangenheit konnte die Zahl der in der Altenpflegeausbildung befindlichen Schülerinnen und Schüler bundesweit um $22 \%$ gesteigert werden ( $\triangleright$ Abschn. 5.2). Dadurch ist es zwar nicht gelungen, die Lücke zwischen dem Fachkräfteangebot und der ungleich schneller wachsenden Fachkräftenachfrage im Altenpflegebereich zu schließen; es ist jedoch anzunehmen, dass auf diese Weise dem Personalengpass in erheblichem Umfang entgegengesteuert werden konnte.

Doch die konkrete Analyse und Bewertung des Ausbaus der besetzten Ausbildungsplätze in der bundesrechtlich geregelten Altenpflegeausbildung ist in der Fachöffentlichkeit durchaus hoch umstritten. Während einige darin den Beweis für eine gestiegene Attraktivität des Altenpflegeberufs sehen, die jüngst beschlossene generalistische Ausbildungsreform am liebsten zurücknehmen und am Status quo des Altenpflegeberufs festhalten würden, halten andere den Altenpflegeberuf trotz der gestiegenen Ausbildungszahlen für ein Auslaufmodell und sehen im Ausbau der Ausbildungsplätze ein trügerisches Ergebnis, das durch Abstriche bei der Qualität der Ausbildung teuer erkauft wurde.

Der Beitrag stellt den erfolgreichen Ausbau der Ausbildungszahlen in der Altenpflege in den Mittelpunkt der Analyse und soll zum besseren Verständnis der ihm zugrunde liegenden Entwicklungen und Dynamiken beitragen. Er schildert, welche spezifischen Strategien beim Ausbau von Ausbildungskapazitäten besonders wirksam waren, aber auch, inwiefern in diesem Zusammenhang Sorgen um die Qualität der Altenpflegeausbildung berechtigt sind.

Dazu nimmt er neben der Umschulungsförderung in der Altenpflege durch die Bundesagentur für Arbeit („Weiterbildung Ge- 
Tabelle 5.1 Schülerzahlen in den dreijährigen Pflegeausbildungsberufen in Deutschland insgesamt. (Statistisches Bundesamt 2012, 2014a, 2014b, 2015, 2017, 2018a, 2018b; eigene Berechnung und Darstellung)

\begin{tabular}{|l|l|l|l|l|l|l|l|l|}
\hline & $2011 / 12$ & $2012 / 13$ & $2013 / 14$ & $2014 / 15$ & $2015 / 16$ & $2016 / 17$ & $2017 / 18$ & Trend \\
\hline Altenpflege & 55.966 & 59.365 & 62.385 & 66.285 & 68.051 & 68.260 & 68.236 & $+22 \%$ \\
\hline $\begin{array}{l}\text { Krankenpflege } \\
\text { Ka.857 }\end{array}$ & $-^{\mathrm{a}}$ & 64.009 & 64.022 & 63.611 & 64.258 & 63.707 & $+6 \%$ \\
\hline $\begin{array}{l}\text { Kinderkranken- } \\
\text { pflege }\end{array}$ & 6.442 & $-^{\mathrm{a}}$ & 6.780 & 6.928 & 7.074 & 7.155 & 7.481 & $+16 \%$ \\
\hline
\end{tabular}

a Das Statistische Bundesamt weist für das Schuljahr 2012/13 keine differenzierten Schülerzahlen für die Berufe Krankenpflege und Kinderkrankenpflege aus

Pflege-Report 2019

ringqualifizierter und beschäftigter älterer Arbeitnehmer in Unternehmen“ - kurz „WeGebAU“) ausgewählte bundeslandspezifische Aktivitäten in den Blick, und zwar in den beiden Bundesländern, die jüngst die mit Abstand höchsten Zuwächse bei der Zahl der Altenpflege-Auszubildenden verzeichnen konnten: Nordrhein-Westfalen und Hessen. Abschließend werden die Befunde im Kontext der 2020 in Kraft tretenden Ausbildungsreform auf Grundlage des Pflegeberufegesetzes diskutiert.

\subsection{Entwicklung der Ausbildungszahlen im Altenpflegebereich}

Zwischen den Schuljahren 2011/12 und 2017/18 ist die Zahl aller in der Altenpflegeausbildung befindlichen Schülerinnen und Schüler von 55.966 auf $68.236(+22 \%)$ angewachsen. In der Krankenpflegeausbildung ist die Zahl der Auszubildenden im gleichen Zeitraum hingegen lediglich um rund $6 \%$ gestiegen. Damit hat die Altenpflegeausbildung - gemessen an der Gesamtzahl der in der Ausbildung befindlichen Personen - die Krankenpflege überholt und ist mit dem Schuljahr 2014/2015 zum zahlenstärksten Ausbildungsberuf im Gesundheitswesen aufgestiegen (•Tab. 5.1).
Der Anstieg der Ausbildungszahlen dauerte in der Altenpflege bis zum Schuljahr 2015/16 an. Seitdem stagniert auch die Zahl aller in einer Altenpflegeausbildung befindlichen Schülerinnen und Schüler.

Die meisten Auszubildenden in der Altenpflege werden in Nordrhein-Westfalen qualifiziert (18.854 im Schuljahr 2017/18), gefolgt von Baden-Württemberg (9.776) und Bayern (7.429). Auf dem vierten Platz befindet sich Niedersachsen (7.269), dahinter Sachsen (4.526) und Hessen (4.187). In diesen sechs Bundesländern wurden im Schuljahr 2017/18 insgesamt 52.041 Auszubildende und damit $76 \%$ aller bundesweit in Ausbildung befindlichen Personen qualifiziert (• Abb. 5.1).

Betrachtet man die Entwicklung in diesen sechs ausbildungsstärksten Bundesländern genauer, so fällt auf, dass sich die Auszubildendenzahlen äußerst unterschiedlich verändert haben. Während Nordrhein-Westfalen und Hessen zwischen den Schuljahren 2012/13 ${ }^{1}$ und 2017/18 die mit Abstand höchsten Steigerungsraten $(+31 \%$ und $+29 \%)$ erzielten, fällt die Bilanz in den restlichen Bundes-

\footnotetext{
Für das Schuljahr 2011/12 und früher weist das Statistische Bundesamt (2012) keine separaten Angaben für Baden-Württemberg, Hessen, Niedersachsen und Sachsen aus. Daher ist ein bundeslandspezifischer Vergleich der Zuwächse bei den Ausbildungskapazitäten ab diesem Jahr nicht möglich.
} 


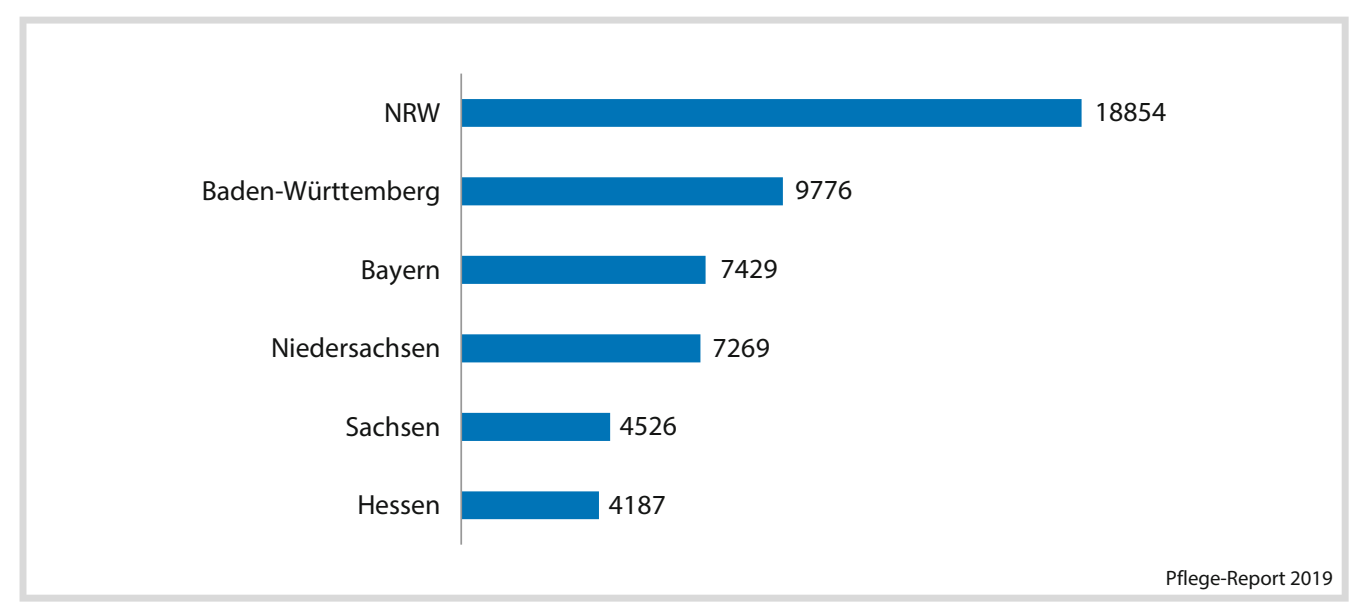

- Abb. 5.1 Bundesländer mit den höchsten Schülerzahlen in der Altenpflegeausbildung im Schuljahr 2017/18. (Statistisches Bundesamt 2018a; eigene Darstellung)

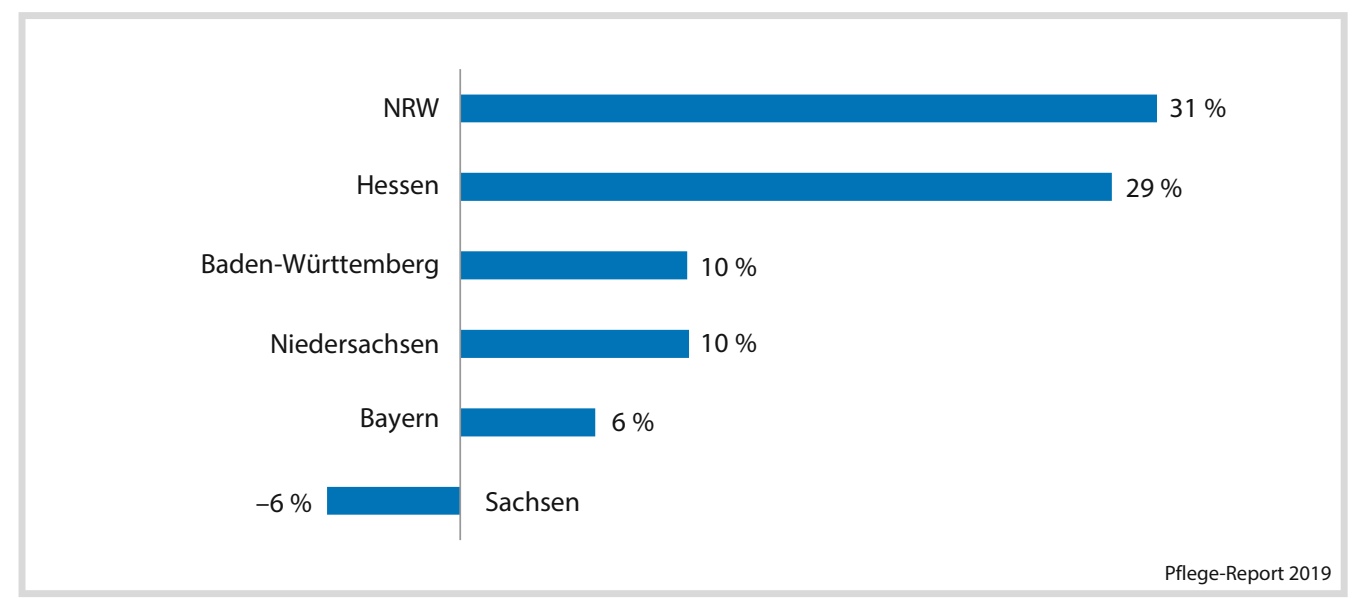

- Abb. 5.2 Entwicklung der Ausbildungskapazitäten in den sechs ausbildungsstärksten Bundesländern zwischen Schuljahr 2012/13 und 2017/18 in der Altenpflege. (Statistisches Bundesamt 2014b, 2018a; eigene Berechnung und Darstellung)

ländern deutlich ernüchternder aus (siehe - Abb. 5.2).

Weitere Angaben zur Größenordnung und Entwicklung der Ausbildungszahlen in den dreijährigen Pflegeberufen liegen auf Bundesebene nicht vor. Insbesondere bleibt unklar, wie viele Auszubildende bundesweit die Alten-, Kinder- und Krankenpflegeausbildung oder ei- ne der Helferausbildungen in jedem Jahr erfolgreich abschließen bzw. wie viele Ausbildungsabbrüche jeweils zu verzeichnen sind. Weitere relevante Daten wie die Anzahl der Schulen, die Struktur des Schulpersonals oder die Entwicklung der praktischen Ausbildungskapazitäten sind auf Bundesebene ebenfalls nicht vorhanden. 


\subsection{Maßnahmen zur Gewinnung neuer Auszubildender in der Altenpflegeausbildung}

\subsubsection{WeGebAU-Förderung der Bundesagentur für Arbeit}

Eine der wesentlichen Initiativen zur Förderung neuer Ausbildungsplätze in der Altenpflege war die „Ausbildungs- und Qualifizierungsoffensive Altenpflege“, die zwischen Bund, Ländern und Verbänden vereinbart und zwischen 2012 und 2015 durch eine Reihe von Maßnahmen umgesetzt worden ist. Das Programm wurde unter der Federführung des für die Altenpflegeausbildung zuständigen Bundesministeriums mit zahlreichen Behörden, Leistungserbringern, Gewerkschaften, Pflegeverbänden und weiteren Stellen erarbeitet und sollte aus Sicht der Beteiligten als konzertierte Aktion entscheidend zur "Sicherung der Fachkraftbasis" im Altenpflegebereich beitragen (siehe Vereinbarungstext 2012).
Eine der wenigen rechtlich verbindlichen und zugleich wichtigsten Fördermaßnahmen ist die (Wieder-)Einführung der dreijährigen Nachqualifizierung/Umschulung in der Altenpflege durch die Bundesagentur für Arbeit (kurz „WeGebAU“). Die Förderung wurde zunächst bis 2015 zeitlich befristet, später auf den Zeitraum bis zur Umsetzung des Pflegeberufegesetzes ab 2020 erweitert.

Zielgruppe der Förderung sind geringqualifizierte Beschäftigte. Die Förderung besteht zum einen darin, dass die Lehrgangskosten durch die Bundesagentur erstattet werden und zum anderen, dass für die gesamte Dauer der Ausbildung (bis zu drei Jahre) die Bundesagentur einen Zuschuss zum Arbeitsentgelt während der Ausbildungszeit finanziert, der sich an dem bisherigen Gehalt orientiert.

Die Wiedereinführung der dreijährigen WeGebAU-Förderung ging mit einem außerordentlichen Effekt einher: Die Zahl der jährlichen Eintritte von WeGebAU-geförderten Personen in die Altenpflegeausbildung stieg sprunghaft um $87 \%$ an. Waren 2012 - also im Vorjahr des Förderbeginns - noch knapp

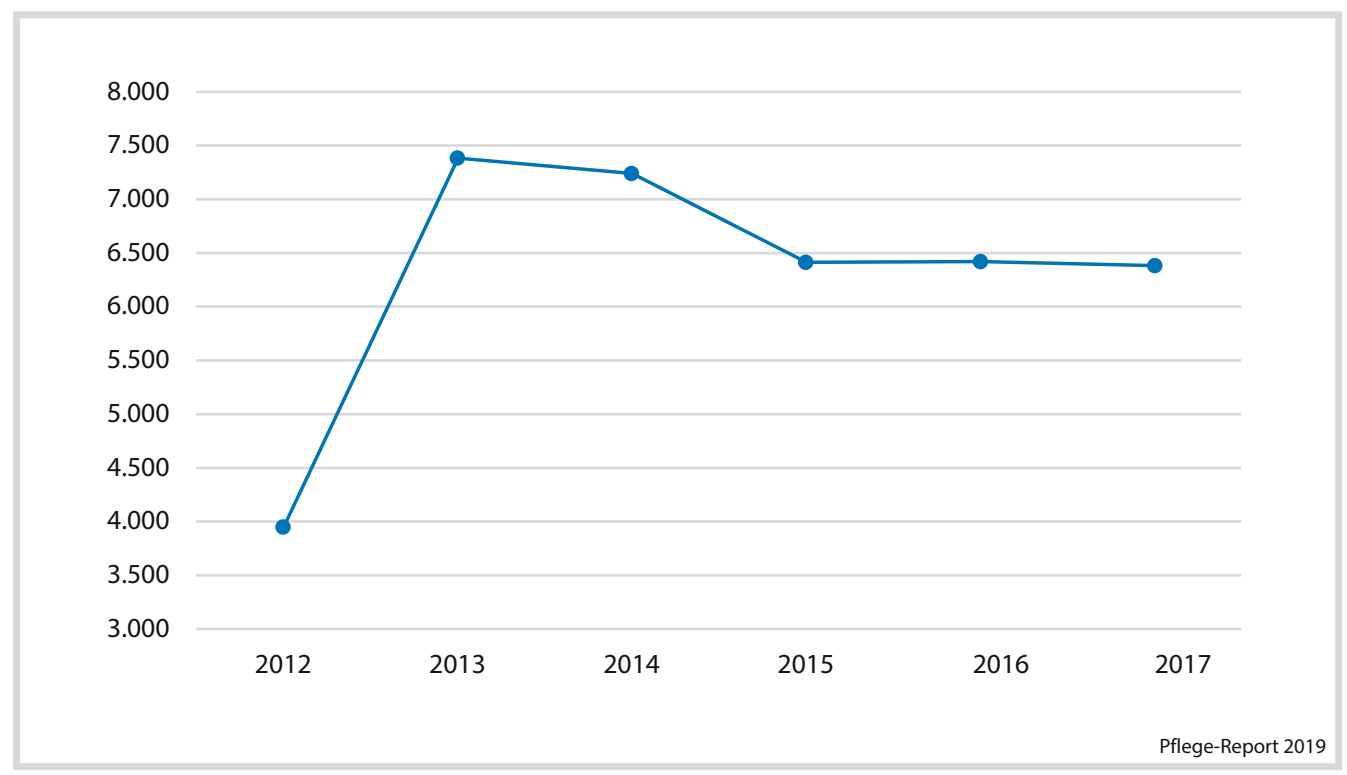

- Abb. 5.3 Zahl der an der WeGebAU-Förderung neu teilnehmenden Personen in der Altenpflegeausbildung. (Bundesagentur für Arbeit 2013, 2014, 2015, 2016, 2017, 2018b; eigene Darstellung) 
3.950 neue Eintritte in die von einer Agentur für Arbeit geförderte Altenpflegeausbildung gemeldet, wuchs die Zahl der Neueintritte im darauffolgenden Jahr auf 7.383. Seit 2013 nahm die Zahl der geförderten Ausbildungseintritte allerdings wieder ab auf 6.382 Neueintritte im Jahr 2017 (• Abb. 5.3).

Seit Einführung der dreijährigen WeGebAU-Förderung ist der Anteil der durch die Arbeitsagentur geförderten Ausbildenden insgesamt angewachsen - von ca. $18 \%$ zwischen 2013/14 auf $22 \%$ in den Jahren 2015 und 2016. Im Jahr 2017 ist ihr Anteil wieder auf knapp $21 \%$ leicht zurückgegangen.

\subsubsection{Bundeslandspezifische Ansätze}

\section{- Nordrhein-Westfalen (NRW)}

In NRW werden deutschlandweit betrachtet mit Abstand die meisten Personen in der Altenpflege ausgebildet. Auch die Zuwächse bei den Ausbildungszahlen in dem Bundesland sind mit rund $31 \%$ zwischen 2012/13 und 2017/18 außerordentlich hoch ausgefallen ( Abschn. 5.2). Weitet man den Betrachtungszeitraum auf das Schuljahr 2011/12 aus, so fällt das im bundesweiten Vergleich überdurchschnittliche Wachstum der Ausbildungsplätze in NRW mit $51 \%$ nochmals stärker aus ${ }^{2}$.

Der hohe Anstieg der besetzten Ausbildungsplätze fiel zeitlich mit der im Jahr 2012 seitens der Landesgesetzgebung eingeführten Umlagefinanzierung der Fachkraftausbildung in der Altenpflege zusammen. Das in NRW bis heute bestehende Umlageverfahren beruht auf einer Fondfinanzierung der durch die Ausbildung entstehenden Kosten bei den ausbildenden Betrieben. Die Beiträge zur Finanzierung des Fonds sind von allen Pflegeheimen und am-

2 Für das Schuljahr 2011/12 weist das Statistische Bundesamt (2012) keine separaten Angaben für BadenWürttemberg, Hessen, Niedersachsen und Sachsen aus. Daher ist ein bundeslandspezifischer Vergleich der Zuwächse bei den Ausbildungskapazitäten ab diesem Jahr nicht möglich. bulanten Pflegediensten aufzubringen, gleichgültig, ob der einzelne Betrieb selbst ausbildet. Auf diese Weise sollen ausbildende Betriebe an dieser Stelle keinen Kosten- beziehungsweise Wettbewerbsnachteil haben.

Während der Phase der Erhöhung der Ausbildungszahlen ist der Anteil der Hauptschülerinnen und Hauptschüler in der Altenpflegeausbildung von 14,5\% im Jahr 2008 auf $35 \%$ im Jahr 2017 angewachsen. Zum Vergleich: In der Krankenpflegeausbildung lag der Anteil der Hauptschülerinnen und Hauptschüler in NRW im Jahr 2008 bei 1,5\% und im Jahr 2017 bei 2,5 \% (MAGS NRW 2010; Landtag NRW 2017; hier eigene Berechnung).

Erwähnenswert ist in diesem Zusammenhang, dass in NRW die allgemeine Schulpflicht zehn Jahre dauert. Hauptschulabsolventinnen und -absolventen aus NRW können - anders als Hauptschulabsolventinnen und -absolventen aus Bundesländern mit einem neunjährigen Schulbesuch - insofern direkt in die Altenpflegeausbildung einmünden.

Angestiegen ist zwischen 2012 und 2018 daneben die Zahl der WeGebAU-geförderten Ausbildenden in der Altenpflege, von 913 Neueintritten im Jahr 2012 auf 1.222 und damit um $34 \%$ im Jahr 2017 (Bundesagentur für Arbeit 2013, 2018b; eigene Berechnung). Damit lag das Wachstum bei den WeGebAU-geförderten Ausbildenden in NRW unterhalb des Bundesdurchschnitts von $87 \%$ ( $>$ Abschn. 5.3.1). Auch mit Blick auf den prozentualen Anteil aller WeGebAU-geförderten Ausbildenden an der Gesamtzahl aller Auszubildenden in der Altenpflege im Jahr 2017 ist die Situation in NRW (12\%) im Bundesvergleich (21\%; - Abschn. 5.3.1) unterdurchschnittlich geblieben.

Unabhängig von Finanzierungsfragen fällt auf, dass die Ausweitung der Ausbildungszahlen in NRW in einer Phase stattfand, in der landesweite Qualitätsvorgaben für die Altenpflegeschulen seitens der nordrhein-westfälischen Landesregierung über einen Zeitraum von mehreren Jahren ausgesetzt wurden. So fehlten bspw. Standards bei der Mindestanzahl und Qualifikation der unterrichtenden Lehrkräfte. 
Es war im weiteren Verlauf die Landesregierung selbst, die das Fehlen dieser Qualitätsvorgaben als Problem eingeräumt und eine (Wieder-)Einführung verbindlicher Qualitätsstandards auf den Weg gebracht hat (Landtag NRW 2014).

Last but not least: Die Steigerung der Ausbildungszahlen in Nordrhein-Westfalen wurde darüber hinaus von vielfältigen Aktionen und Kampagnen zur Image-Förderung bzw. zur Steigerung des Interesses für eine Ausbildung begleitet, an denen neben der Landesregierung unterschiedliche Partner beteiligt waren. Gefördert wurden außerdem verschiedene Projekte zur Erprobung und Förderung spezifischer Ausbildungsformate (z. B. Teilzeitkurse) bzw. zur gezielten Erschließung neuer Zielgruppen (z. B. Migranten/Geflüchtete).

\section{- - Hessen}

Hessen weist im Zeitraum zwischen 2012/13 und 2017/18 innerhalb der Gruppe der sechs ausbildungsstärksten Bundesländer knapp hinter NRW die zweithöchsten Zuwächse an besetzten Ausbildungsplätzen im Altenpflegeberuf aus (+29\%; Abschn. 5.2).

Ebenfalls ähnlich wie in NRW wurde der hessische Kapazitätsausbau von zahlreichen Initiativen und Projekten zur Werbung von Auszubildenden begleitet. Auch in Hessen wurden Zielgruppen angesprochen, die bisher entweder von einer Ausbildung ausgeschlossen waren oder diese aufgrund bestimmter persönlicher Eigenschaften nur unter großen Schwierigkeiten hätten bewältigen können. Zu nennen wäre bspw. die Einführung einer landesfinanzierten Sprachförderung in der Altenpflegeund Altenpflegehilfeausbildung für Personen mit unzureichenden deutschen Sprachkenntnissen.

Ebenfalls ähnlich wie in NRW verfügt ungefähr jeder Dritte der hessischen Altenpflegeauszubildenden über einen Hauptschulabschluss. Auch in Hessen entspricht das einem für Gesundheitsberufe überproportional hohen Anteil (HSL 2015).

Darüber hinaus unterscheiden sich die Maßnahmen und Begleitumstände zur Gewinnung zusätzlicher Auszubildender in Hessen an zahlreichen Punkten verhältnismäßig deutlich von denen in NRW. Beispielsweise existiert in Hessen keine Umlagefinanzierung der Fachkraftausbildung. In Hessen ging der Anstieg der Zahl der Auszubildenden zwischen 2012 und 2017 auch nicht auf einen entsprechenden Zuwachs an regulär finanzierten Ausbildungsplätzen zurück, sondern kam in erster Linie durch eine rekordhafte Zunahme der durch die Bundesagentur für Arbeit geförderten WeGebAUAusbildungsplätze zustande (+273\% bei Neueintritten; vgl. - Tab. 5.2). Hessen sticht an dieser Stelle nicht nur im direkten Vergleich mit NRW außergewöhnlich deutlich heraus (NRW $+34 \%$ ), sondern stellte auch im Bundesvergleich (+87\% im Bund; - Abschn. 5.3.1) einen Rekord auf. Diese Entwicklung hatte hinsichtlich der verschieden aufgestellten Finanzierung der Ausbildungsplätze zur Folge, dass im Jahr 2017 in Hessen bereits beinahe jeder dritte

\begin{tabular}{|c|c|c|c|c|c|c|c|}
\hline & 2012 & 2013 & 2014 & 2015 & 2016 & 2017 & Trend \\
\hline $\begin{array}{l}\text { Landesfinanzierte } \\
\text { Ausbildungsplätze }^{a}\end{array}$ & 1.200 & 1.375 & 1.105 & 1.235 & 1.267 & 1.228 & $+2 \%$ \\
\hline $\begin{array}{l}\text { WeGebAU-geförderte } \\
\text { Ausbildungsplätze }\end{array}$ & 135 & 282 & 377 & 395 & 439 & 503 & $+273 \%$ \\
\hline
\end{tabular}


neue Auszubildende in der Altenpflege von der Bundesagentur für Arbeit gefördert war (- Tab. 5.2).

Betrachtet man demgegenüber ausschließlich die Neueintritte in die regulär finanzierte Altenpflegeausbildung, so ist festzustellen, dass die Zahl der Auszubildenden zwischen 2012 (1.200) und 2017 (1.228) mehr oder weniger unverändert geblieben ist (•Tab. 5.2).

In Hessen werden außerdem überproportional viele Personen im Rahmen der einjährigen landesrechtlich geregelten Altenpflegehilfeausbildung ausgebildet. Im Schuljahr 2017/18 kam in Hessen auf vier Auszubildende in der Altenpflege ein Auszubildende/ $r$ in der Altenpflegehilfe. Im Bundesdurchschnitt kommen hingegen acht Auszubildende in der Altenpflege auf eine/n Auszubildende/n im Altenpflegehilfebereich. In NRW liegt der Anteil sogar bei rund $16 \mathrm{zu} 1$ (Statistisches Bundesamt 2018a; hier eigene Berechnung).

Aufgrund der großen Kapazitäten in der Altenpflegehilfe wird der Zielgruppe der Hauptschulabsolventinnen und -absolventen mit neunjähriger Schulbildung systematisch und in hohen Umfängen ein Zugang zur Altenpflegeausbildung ermöglicht. Anders als in NRW besteht in Hessen nämlich keine allgemeine zehnjährige Schulpflicht, sodass der Weg über die Altenpflegehilfe erforderlich ist, um Hauptschülerinnen und Hauptschüler zu gewinnen. Ungefähr jede zweite Absolventin bzw. jeder zweite Absolvent der hessischen Altenpflegehilfe nimmt an einer in der Regel um ein Jahr verkürzten Fachkraftausbildung teil (RP 2016).

Der Ausbau der Ausbildungskapazitäten fand in Hessen auf der Grundlage von vergleichsweise niedrigen landesrechtlich geregelten Qualitätsstandards statt. Während bspw. in NRW nach (Wieder-)Einführung gesetzlicher Vorgaben von einer hauptberuflichen Lehrkraft eine Hochschulqualifikation - idealerweise ein Master- oder Lehramtsabschluss - gefordert wird, ist in Hessen kein akademischer Abschluss notwendig, sondern der Besuch einer 400-stündigen beruflichen Weiterbildung ausreichend (RP 2011). Zudem gilt in Hessen die
Lehrkraftausstattung an Altenpflegeschulen gemäß $\$ 15$ (2) Hessische Verordnung zur Altenpflege auch dann noch als ausreichend, wenn auf eine Vollzeit-Lehrkraftstelle zwei Ausbildungskurse und damit bis zu 60 Schülerinnen und Schüler entfallen - eine im Ländervergleich unübliche Definition einer Lehrermindestbesetzung.

\subsection{Diskussion}

- - Große Hebelwirkung: Nachqualifizierung Ungelernter und Umlagefinanzierung Einen entscheidenden Anteil an den bisherigen Wachstumsraten im AltenpflegeAusbildungssektor hatte die (Wieder-)Einführung der dreijährigen Umschulungsmaßnahme der Bundesagentur für Arbeit (WeGebAU). Durch die Maßnahme können vor allem viele ältere Personen für eine Fachkraftausbildung gewonnen werden, die als ungelernte Beschäftigte („Hilfskräfte“ ohne staatlich anerkannten Abschluss in einem Pflegeberuf) in Pflegeeinrichtungen häufig seit vielen Jahren tätig sind.

Das spezifische Förderangebot für Zielgruppen, die in vielen Fällen aus bildungsfernen Milieus kommen bzw. migrationsbedingt über keine anerkannten Qualifikationsabschlüsse verfügen, stellt für die betroffenen Personen häufig die berufsbiografisch erste und letzte Chance auf einen beruflichen Fachabschluss dar (Grgic et al. 2018).

Bei der Umsetzung der WeGebAUMaßnahme zwischen den Bundesländern existieren jedoch erhebliche Unterschiede bei den Förderquoten. Die Frage nach den Gründen und damit womöglich nach ungenutzten Potenzialen zur Nachqualifizierung Ungelernter drängt sich auf, kann jedoch auf Basis der vorliegenden Daten nicht beantwortet werden.

Die in NRW eingeführte Umlagefinanzierung der Fachkraftausbildung in der Altenpflege erwies sich als außerordentlich wirkungsvoll, allerdings verbunden mit einem deutlichen Anstieg des Anteils von Personen mit 
Hauptschulabschluss in der Altenpflegeausbildung.

\section{- Fundament des Kapazitätsausbaus: Hauptschulabsolventinnen und -absolventen}

Die bundesweit gestiegene Nachfrage nach Ausbildungsplätzen in der Altenpflegeausbildung ist auf eine im Vergleich $\mathrm{zu}$ den anderen bundesrechtlich geregelten Pflege- und Gesundheitsfachberufen beispiellose Öffnung der Altenpflegeausbildung für Hauptschulabsolventinnen und Hauptschulabsolventen zurückzuführen. Die Wege zur Erschließung dieser Zielgruppe fallen unterschiedlich aus, die Resultate sind vergleichbar. In NRW und Hessen, den Bundesländern mit den höchsten $\mathrm{Zu}-$ wächsen bei den Ausbildungszahlen, besitzt inzwischen ungefähr jede dritte in der Altenpflegeausbildung befindliche Person einen Hauptschulabschluss. Derartig hohe Anteile von Hauptschulabsolventinnen und Hauptschulabsolventen weist kein anderer bundesrechtlich geregelter Gesundheitsfachberuf auf, auch nicht die Gesundheits- und Krankenpflege.

Die in die Reformdiskussion um die Pflegeausbildung eingebrachte Forderung, die schulischen Zugangsvoraussetzungen zur Aufnahme einer Pflegeausbildung zu erhöhen, hätte im Falle ihrer Umsetzung besonders für die Altenpflege gravierende Konsequenzen gehabt. Jedenfalls ist die Befürchtung plausibel, dass dadurch Ausbildungsplätze im zweistelligen Prozentbereich gefährdet würden.

\section{- - Die „Kehrseite“ des Kapazitätsausbaus: Heterogene Qualitätsstandards}

Die Steigerung der Ausbildungszahlen in der Altenpflege hat unter Rahmenbedingungen stattgefunden, die keine vollständige Gewähr für eine verlässliche und im Niveau angemessene schulische Qualitätssicherung geben konnten.

Als problematisch erscheinen die erheblichen Unterschiede bei den bundeslandspezifischen schulischen Qualitätsvorgaben. Die nordrhein-westfälische Landesregierung hat- te ihre landesrechtlichen Mindestanforderungen an die schulische Ausbildung im Zuge des Ausbaus der Ausbildungsplätze zwischenzeitlich ausgesetzt, in Hessen liegen diese teilweise deutlich unter dem Niveau anderer Bundesländer. Diese Liste der unterschiedlichen Mindestanforderungen insbesondere hinsichtlich der Personalausstattung an Schulen kann um weitere Beispiele erweitert werden (Lauxen und Slotala 2015; Sahmel 2018; Tegethoff et al. 2016).

Eine schon immer bestehende Lücke in der Qualitätssteuerung, die allerdings nicht erst im Zusammenhang mit den jüngst verstärkten Bemühungen um zusätzliche Auszubildende entstanden ist, sondern dem Altenpflegegesetz seit seiner Einführung innewohnt, ist im Zusammenhang mit der Durchführung der staatlichen Abschlussprüfung zu finden. Die staatliche Abschlussprüfung in der Altenpflege kann auch in den Fällen bestanden werden, in denen insgesamt mangelhafte oder ungenügende Prüfungsleistungen erbracht werden. Aufgrund der im Prüfungsrecht, $\$ 9$ der Ausbildungsund Prüfungsverordnung für den Beruf der Altenpflegerin und des Altenpflegers, gegebenen Möglichkeit des Notenausgleichs können die im Staatsexamen erzielten Noten 5 und 6 durch Vornoten, die im Verlauf der Ausbildung vergeben wurden, ausgeglichen werden. Eine vergleichbare Regelung findet in keinem anderen Pflege- oder Gesundheitsfachberuf Anwendung.

Insofern ist bei den verstärkten Bemühungen zur Gewinnung leistungsschwächerer Zielgruppen für die Altenpflegeausbildung auch zu berücksichtigen, dass die staatliche Abschlussprüfung nur eingeschränkt eine nicht ausreichende Pflegekompetenz konsequent sanktionieren kann.

\section{- Bemerkenswert: Bildungssteuerung ohne Bildungsberichterstattung}

Die wohl wichtigste Frage ist, inwiefern die zwischen den Schuljahren 2011/12 und 2017/18 bundesweit erfolgte Steigerung von Ausbildungszahlen tatsächlich einen signifikanten Beitrag zur Abmilderung der Fachkräfteeng- 
pässe im Altenpflegebereich leisten konnte. Die Antwort darauf lautet: Wir wissen es nicht genau.

Eine der eklatantesten Wissenslücken betrifft die jährliche bundesweite Anzahl der Personen, die eine Altenpflegeausbildung erfolgreich abschließen konnten und als Altenpflegefachkraft dem Arbeitsmarkt neu zur Verfügung standen. Niemand erfasst zuverlässig und für das gesamte Bundesgebiet, ob die neuen für die Ausbildung gewonnenen Zielgruppen, wie ältere Personen oder Hauptschulabsolventinnen und Hauptschulabsolventen, die Altenpflegeausbildung meistern. Wie die Pflegeschulen personell aufgestellt sind, mit welchem zusätzlichen Lehrerbedarf aufgrund des notwendigen Ausbaus der Ausbildungsplätze zu rechnen ist - für eine kontinuierliche Ermittlung derartiger Informationen ist ebenfalls niemand zuständig.

Die bisherige Planung und Umsetzung von Maßnahmen zur Gewinnung neuer Auszubildender basiert auf rudimentär vorhandenen Daten. Eine umfassende und kontinuierliche Bildungsberichterstattung in den Pflegeberufen sieht anders aus (Slotala und Ewers 2012) und wäre gerade vor dem Hintergrund der weiter wachsenden Bedeutung dieses Ausbildungsbereichs für den Arbeitsmarkt dringend nötig.

\subsection{Schlussbetrachtung und Ausblick auf das Pflegeberufegesetz}

Es ist der Altenpflegebranche gelungen, ihre Ausbildungskapazitäten insgesamt zu vergrößern. Durch mehrere Maßnahmen konnten erfolgreich zusätzliche Personen für die Ausbildung gewonnen werden. Als die Stellschrauben mit der größten Wirkung stechen einerseits die Verbesserung der finanziellen Ausbildungsanreize hervor sowie andererseits die Ansprache von ungelernten Hilfskräften sowie von Hauptschülerinnen und Hauptschülern in einem für Gesundheitsfachberufe signifikant hohen Umfang.
Allerdings ist der Anstieg der Ausbildungszahlen im Altenpflegebereich längst nicht in allen Bundesländern in vergleichbarem Umfang gelungen. Zudem sind die Zuwächse bei den Auszubildenden bundesweit seit dem Schuljahr 2015/16 ins Stocken geraten. Ein Grund dürfte in der föderalen Zuständigkeit bei der Finanzierung der Altenpflegeausbildung zu finden sein. Die Konsequenz: Während in NRW im Zuge der neuen Umlagefinanzierung die Ausbildungszahlen signifikant steigen, können sich andere Bundesländer nicht zur Umlagefinanzierung durchringen. Während Hessen jedem potenziellen Auszubildenden einen landesfinanzierten Schulplatz zusichert, werden Ausbildungswillige in anderen Bundesländern mitunter mit der Aufforderung konfrontiert, ein monatliches Schulgeld zu zahlen. Koordinierte und verbindliche Strategien zur Erhöhung der Zahl der Auszubildenden vor dem Hintergrund eines bereits vorhandenen und zukünftig weiter wachsenden Fachkräftemangels im gesamten Bundesgebiet sehen sicherlich anders aus.

Zudem hat die Erhöhung der Ausbildungskapazitäten einen - für einen Gesundheitsfachberuf überaus kritisch zu sehenden - Wermutstropfen: Die Qualitätssicherung der Altenpflegeausbildung befindet sich bis heute auf einem unzuverlässigen Niveau. Insofern muss offenbleiben, ob der Weg des Kapazitätsausbaus zu Lasten der Ausbildungsqualität beschritten wurde.

Die 2020 in Kraft tretende Ausbildungsreform stellt in gewisser Weise eine Zäsur im Pflegebildungssystem dar (Igl 2018). Die Krankenpflegeausbildung wird durch eine neue generalistische Pflegeausbildung ersetzt. Erstmals werden Vorbehaltstätigkeiten für Pflegefachpersonen definiert, neue Finanzierungsgrundlagen geschaffen und formale Rahmenbedingungen eines regelhaften Pflegestudiums verankert.

Ob durch das Pflegeberufegesetz zusätzliche Auszubildendenzahlen im Langzeitpflegebereich generiert werden könnten, kann freilich nur mutmaßlich beantwortet werden. Dafür spricht, dass die Ausbildungsreform an 
einige Maßnahmen zur Erhöhung von Ausbildungsplätzen in der Altenpflege anknüpft, die als erfolgreich identifiziert wurden. Dazu zählt insbesondere die Einführung einer bundesweit einheitlichen Regelungsgrundlage einer Ausbildungsfinanzierung per Umlageverfahren. An dieser Stelle besteht mit Blick auf die positiven Erfahrungen mit der Umlagefinanzierung in NRW ein begründeter Anlass zur Vermutung, dass davon ein signifikanter Impuls zur verstärkten Ausbildungsaktivität im Langzeitpflegesektor ausgehen könnte.

Die mit der neuen Finanzierungsgrundlage im Pflegeberufegesetz einhergehende bundesweite Abschaffung des Schulgeldes dürfte ebenfalls einen weiteren Schub auslösen.

Demgegenüber bleibt abzuwarten, ob sich wie von manchen befürchtet (Dielmann 2019) - die Verbreiterung der praktischen Einsatzgebiete und die damit einhergehende Reduzierung der Präsenzzeiten des einzelnen Auszubildenden im Ausbildungsbetrieb negativ auf die Ausbildungsbereitschaft der Einrichtungen auswirken wird. Zu berücksichtigen ist gleichfalls, dass im Zuge der Umstellung auf das Pflegeberufegesetz zwischen den Trägern der praktischen Ausbildung höchstwahrscheinlich neue Ausbildungs- und Kooperationsverbünde aufgebaut werden, um unter anderem eine ausgeglichene und damit auch faire Rotation von Auszubildenden in den Betrieben sicherzustellen.

Mit dem Pflegeberufegesetz werden die bundesweit gültigen Qualitätsstandards bei der schulischen und praktischen Ausbildung zum Teil deutlich angehoben. Es ist insbesondere in Bundesländern mit vergleichsweise niedrigen Schulqualitätsstandards zu erwarten, dass die Anpassung an höhere Vorgaben wahrscheinlich nicht „einfach“ und „schnell“ vonstattengehen wird. Jedenfalls bedarf es beispielsweise für die Schaffung zusätzlicher akademisch qualifizierter Lehrkräfte auf Masterniveau notwendigerweise mehrjähriger Vorlaufzeiten (Lauxen und Slotala 2015). Der ab 2020 anvisierte Kapazitätsausbau im Altenpflegesektor wird mancherorts deshalb mit ungleich höheren Umsetzungshürden verbunden sein.
Die Altenpflege wird als eigenständiger Berufsabschluss auch im Pflegeberufegesetz erhalten bleiben. Der Bundesgesetzgeber hat kurz vor Verabschiedung des Pflegeberufegesetzes explizit aus Sorge vor einem zu hohen Ausbildungsniveau in der Altenpflege das Kompetenzniveau bei der Abschlussprüfung in diesem Bereich einseitig abgesenkt. Dieser Schritt wird verständlich, wenn man berücksichtigt, dass die Ausbildungszahlen bisher vor allem dadurch gesteigert werden konnten, weil bildungsschwächere Zielgruppen erschlossen wurden.

Ob die im „Last-Minute-Verfahren“ vollzogene einseitige Absenkung des Kompetenzniveaus der Altenpflegeausbildung mittelfristig den erwünschten Effekt zeigen wird oder ob hier ein Korridor beschritten wurde, an dessen Ende der Berufsstatus der Altenpflege im Verhältnis zu den anderen Pflegefachberufen neu austariert werden wird, bleibt ungewiss. Sicher ist: In keiner anderen Pflegebranche werden prognostisch mehr Fachkräfte benötigt als in der Langzeitpflege. Der Druck auf und in dem Ausbildungsbereich, kontinuierlich mehr Auszubildende zu gewinnen, wird noch weiter zunehmen.

\section{Literatur}

Bundesagentur für Arbeit (2013) Teilnehmer in Maßnahmen zur Förderung der beruflichen Weiterbildung (FbW). Arbeitsmarkt in Zahlen, Förderstatistik

Bundesagentur für Arbeit (2014) Teilnehmer in Maßnahmen zur Förderung der beruflichen Weiterbildung (FbW). Arbeitsmarkt in Zahlen, Förderstatistik

Bundesagentur für Arbeit (2015) Teilnehmer in Maßnahmen zur Förderung der beruflichen Weiterbildung (FbW). Arbeitsmarkt in Zahlen, Förderstatistik

Bundesagentur für Arbeit (2016) Teilnehmer in Maßnahmen zur Förderung der beruflichen Weiterbildung (FbW). Arbeitsmarkt in Zahlen, Förderstatistik

Bundesagentur für Arbeit (2017) Teilnehmende in Maßnahmen zur Förderung der beruflichen Weiterbildung (FbW). Arbeitsmarkt in Zahlen, Förderstatistik

Bundesagentur für Arbeit (2018a) Fachkräfteengpassanalyse. Berichte: Blickpunkt Arbeitsmarkt. Juni 2018. Statistik/Arbeitsmarktberichterstattung. https://statistik.arbeitsagentur.de/Statischer- 
Content/Arbeitsmarktberichte/FachkraeftebedarfStellen/Fachkraefte/BA-FK-Engpassanalyse-201806.pdf. Zugegriffen: 30. Nov. 2018

Bundesagentur für Arbeit (2018b) Förderung der beruflichen Weiterbildung. Deutschland, Länder und Regionaldirektionen

Dielmann G (2019) Anmerkungen zur Ausbildungsreform der Pflegeberufe. Dr Med Mabuse 237:45-48

Grgic M, Riedel B, Weihmayer LS, Weimann-Sandig N, Wirner L (2018) Quereinsteigende auf dem Weg zur Fachkraft. Ergebnisse einer qualitativen Studie in den Berufsfeldern Kindertagesbetreuung und Altenpflege. Hans-Böckler-Stiftung, Düsseldorf

Hackmann T, Klein R, Schneidenbach T, Anders M, Vollmer J (2016) Report Pflegeinfrastruktur - Die pflegerische Versorgung im Regionalvergleich. Bertelsmann Stiftung, Gütersloh

HSL - Hessisches Statistisches Landesamt (2015) Statistik der Schulen des Gesundheitswesens - Schuljahr 2014/15

Igl G (2018) Gesetz über die Pflegeberufe (Pflegeberufegesetz - PflBG). Praxiskommentar. medhochzwei, Heidelberg

Kochskämper S (2018) Die Entwicklung der Pflegefallzahlen in den Bundesländern. Eine Simulation bis 2035. IW-Report 33/18. Institut der Deutschen Wirtschaft

LNRW - Landtag Nordrhein-Westfalen (2014) Antwort der Landesregierung auf die Kleine Anfrage 2400 vom 25. Juni 2014 der Abgeordneten Susanne Schneider und Ulrich Alda, FDP. Drucksache 16/6438

LNRW - Landtag Nordrhein-Westfalen (2017) Antwort der Landesregierung auf die Kleine Anfrage 5685 vom 6. März 2017 des Abgeordneten Oskar Burkert, CDU. Drucksache 16/14451

MAGS NRW - Ministerium für Arbeit, Gesundheit und Soziales des Landes Nordrhein-Westfalen (2010) Landesberichterstattung Gesundheitsberufe Nordrhein-Westfalen 2010

Lauxen O, Slotala L (2015) Lehrkräfte an Kranken- und Altenpflegeschulen. Aktuelle Situation und zukünftiger Bedarf. PADUA 10:48-54

Ostwald DA, Ehrhard T, Bruntsch F, Schmidt H, Freidl C (2010) Fachkräftemangel. Stationärer und ambulanter Bereich bis zum Jahr 2030. PricewaterhouseCoopers AG. https://www.pwc. de/de/gesundheitswesen-und-pharma/assets/ fachkraeftemangel.pdf. Zugegriffen: 11. Nov. 2018

Rothgang H, Müller R, Unger R (2012) Themenreport „Pflege 2030". Was ist zu erwarten - was ist zu tun? Bertelsmann Stiftung, Gütersloh. https://www.bertelsmann-stiftung.de/fileadmin/ files/BSt/Publikationen/GrauePublikationen/GP Themenreport_Pflege_2030.pdf. Zugegriffen: 30. Nov. 2018

RP - Regierungspräsidium Darmstadt (2016) Prüfungsstatistik 2015

RP - Regierungspräsidium Darmstadt (2018) Statistik Ausbildungsvergütung Altenpflege 2007

RP - Regierungspräsidium Darmstadt (2011) Durchführung des Altenpflegegesetzes (AltPflG). Pädagogische Qualifikation der hauptberuflichen Lehrkräfte im Rahmen der staatlichen Anerkennung einer Altenpflegeschule in Hessen. Rundverfügung vom 24. März 2011

Sahmel K-H (2018) Die Entwicklung der PflegelehrerBildung in Deutschland - Rückblick und Ausblick. In: Sahmel K-H (Hrsg) Hochschuldidaktik der Pflege und Gesundheitsfachberufe. Springer, Berlin/Heidelberg, S 41-51

Slotala L, Ewers M (2012) Bildungsberichterstattung in der Pflege. Pflege Ges 17(1):63-74

Statistisches Bundesamt (2012) Bildung und Kultur. Berufliche Schulen. Schuljahr 2011/2012. Fachserie 11. Reihe 2

Statistisches Bundesamt (2014a) Bildung und Kultur. Berufliche Schulen. Schuljahr 2013/2014. Fachserie 11. Reihe 2

Statistisches Bundesamt (2014b) Bildung und Kultur. Berufliche Schulen. Schuljahr 2012/2013. Fachserie 11. Reihe 2

Statistisches Bundesamt (2015) Bildung und Kultur. Berufliche Schulen. Schuljahr 2014/2015. Fachserie 11. Reihe 2

Statistisches Bundesamt (2017) Bildung und Kultur. Berufliche Schulen. Schuljahr 2015/2016. Fachserie 11. Reihe 2

Statistisches Bundesamt (2018a) Bildung und Kultur. Berufliche Schulen. Schuljahr 2017/2018. Fachserie 11. Reihe 2

Statistisches Bundesamt (2018b) Bildung und Kultur. Berufliche Schulen. Schuljahr 2016/2017. Fachserie 11. Reihe 2

Tegethoff D, Wild H, Ewers M (2016) Lehrende für die Gesundheits- und (Kinder-)Krankenpflege - Unordnung in den deutschen Bundesländern. Pflegezeitschrift 69(4):1-8

Vereinbarungstext (2012) Ausbildungs- und Qualifizierungsoffensive Altenpflege 2012-2015 
Open Access Dieses Kapitel wird unter der Creative Commons Namensnennung 4.0 International Lizenz (http:// creativecommons.org/licenses/by/4.0/deed.de) veröffentlicht, welche die Nutzung, Vervielfältigung, Bearbeitung, Verbreitung und Wiedergabe in jeglichem Medium und Format erlaubt, sofern Sie den/die ursprünglichen Autor(en) und die Quelle ordnungsgemäß nennen, einen Link zur Creative Commons Lizenz beifügen und angeben, ob Änderungen vorgenommen wurden.

Die in diesem Kapitel enthaltenen Bilder und sonstiges Drittmaterial unterliegen ebenfalls der genannten Creative Commons Lizenz, sofern sich aus der Abbildungslegende nichts anderes ergibt. Sofern das betreffende Material nicht unter der genannten Creative Commons Lizenz steht und die betreffende Handlung nicht nach gesetzlichen Vorschriften erlaubt ist, ist für die oben aufgeführten Weiterverwendungen des Materials die Einwilligung des jeweiligen Rechteinhabers einzuholen.

(c) (i) 